\title{
Классические звезды типа Т Тельца: аккреция, ветер, пыль
}

\author{
П.П. Петров \\ ФГБУН "Крымская астрофизическая обсерватория РАН”, Научный, Крым, 298409 \\ petrov@craocrimea.ru
}

Поступила в редакцию 23 сентября 2020 г.

\begin{abstract}
Аннотация. Классические звезды типа Т Тельца (CTTS) находятся в ранней фазе эволюции, когда звезда окружена аккреционным диском. Большинство наблюдаемых явлений активности CTTS обусловлены процессами магнитосферной аккреции и ветра. В статье приводятся примеры наблюдений магнитосферной аккреции и образования эффекта вуалирования фотосферного спектра. Показано, что пыль, выносимая ветром с поверхности аккреционного диска, ответственна за наблюдаемую нерегулярную переменность блеска CTTS. В Крымской астрофизической обсерватории с 2013 г. проводится спектральный и фотометрический мониторинг двух звезд, RY Tau и SU Aur, с целью исследования динамики аккреции и ветра на временной шкале от нескольких суток до нескольких лет. Наблюдаемые изменения динамики газовых потоков могут быть вызваны как изменением темпа аккреции, так и изменением глобального магнитного поля молодой звезды.
\end{abstract}

Ключевые слова: молодые звезды, RY Tau, SU Aur, RW Aur

\section{1 Введение}

Звезды типа Т Тельца впервые были исследованы и выделены в новый тип переменных 75 лет назад (Јоу, 1945). Среди отличительных признаков - нерегулярная переменность блеска, эмиссионный спектр низкого возбуждения и связь с темными газопылевыми облаками. Первым, кто пришел к выводу об эволюционной молодости этих звезд, был В.А. Амбарцумян (1949).

В настоящее время классическими звездами типа Т Тельца (CTTS) принято называть молодые звезды малых масс $\left(\leq 2 \mathrm{M}_{\odot}\right)$ с аккреционными дисками. Время существования аккреционного диска составляет в среднем около десяти миллионов лет. После формирования планетной системы и диссипации аккреционного диска звезда становится менее активной и переходит в класс так называемых "Weak-line TTS" (WTTS). Основные сведения о звездах типа Т Тельца можно найти в обзорах: Petrov (2003), Bouvier et al. (2007), Hartmann et al. (2016).

В отличие от более массивных $\left(>2 \mathrm{M}_{\odot}\right)$ молодых звезд, относящихся к классу Ае/Ве звезд Хербига (НАеВе), CTTS имеют конвективные оболочки и, как следствие, более сильные магнитные поля и выраженную активность "магнитного типа", в частности, рентгеновское излучение (Pevtsov et al., 2003). Это существенное отличие, поскольку взаимодействие аккреционного диска с магнитным полем звезды порождает большинство наблюдаемых проявлений активности CTTS.

Современные представления о физических процессах, происходящих на звездах типа Т Тельца, основаны на модели магнитосферной аккреции. Эта модель была разработана для нейтронных звезд (Ghosh and Lamb, 1979) и позже применена к звездам типа Т Тельца (Camenzind, 1990; Koenigl, 1991). В отличие от компактных объектов, где время падения вещества измеряется миллисекундами (нейтронные звезды) или минутами (белые карлики в составе катаклизмических переменных), на CTTS это время составляет около суток. Это позволяет наблюдать движение газовых потоков с достаточным временным разрешением и проводить детальное сравнение наблюдаемых явлений с тем, что дает численное моделирование магнитогидродинамических (МГД) процессов. 
Таблица 1. Спектральные типы CTTS

\begin{tabular}{lll}
\hline Звезда & Спектр & Ссылка \\
\hline SU Aur & G2 III & Herbig and Bell (1988) \\
V1331 Cyg & G7-K0 IV & Petrov et al. (2014b) \\
RY Tau & K1 IV & Herbig and Bell (1988) \\
RW Aur A & K1 & Herbig and Bell (1988) \\
RW Aur B & K5 IV & Dodin et al. (2020) \\
S CrA SE & K6 IV & Gahm et al. (2018) \\
DR Tau & K7 V & Petrov et al. (2011) \\
\hline
\end{tabular}

В данной статье дан краткий обзор результатов наблюдений аккреции и ветра избранных CTTS, полученных с участием автора в разные годы, и представлены новые результаты исследований, проводимых в КрАО РАН. Спектральные типы звезд, обсуждаемых в данной статье, приведены в табл. 1.

\section{2 Аккреция и вуалирование фотосферного спектра}

Наблюдения на интерферометре ALMA в миллиметровом диапазоне длин волн впервые дали возможность получить изображения околозвездных дисков большого числа CTTS, определить их размеры, массы, угол наклона, увидеть признаки планетообразования в структуре этих дисков (Dong et al., 2018; Long et al., 2019). Самые внутренние области аккреционных дисков наблюдались ранее на интерферометрах в ближнем ИК-диапазоне. Размеры внутренней границы пылевого диска CTTS составляют 0.05-0.2 a.e. (Akeson et al., 2005). Магнитосферы CTTS имеют меньшие размеры и пространственно пока неразрешимы. Тем не менее, можно определить направление и лучевые скорости газовых потоков внутри магнитосферы и в ветре по профилям спектральных линий.

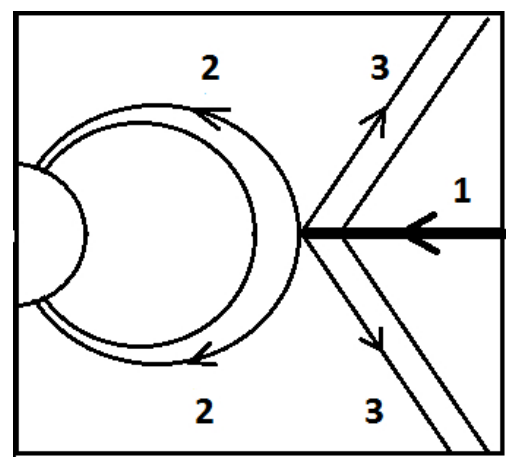

Рис. 1. Основные направления потоков аккреции и ветра: 1 - дисковая аккреция, 2 - магнитосферная аккреция, 3 - ветер

На рис. 1 схематично показаны направления потоков аккреции и ветра в модели магнитосферной аккреции. При достаточно большом угле наклона оси магнитного диполя луч зрения пересекает как область ветра, так и область магнитосферной аккреции. На рис. 2 приведены профили бальмеровских линий водорода в спектре S CrA SE (Gahm et al., 2018).

Типичный профиль эмиссионной линии $\mathrm{H}_{\alpha}$ в спектре CTTS имеет депрессию в “синем" крыле, вызванную поглощением в ветре. Это профиль типа В II-III по классификации Reipurt et al. (1996). Поглощение (ниже континуума) в падающем потоке газа хорошо заметно в высоких членах бальмеровской серии, где эмиссия не столь интенсивна. Хорошими индикаторами аккреции являются линии водорода в ИК-диапазоне: $\mathrm{Pa} \beta, \mathrm{Br} \gamma$ (Folha and Emerson, 2001), а также линия Не I $10800 \AA$. В видимой области спектра обратный Р Суg профиль обычно виден в линии Не I $5886 \AA$, в линиях резонансного дублета D Na I и в линиях триплета О г $7773 \AA$.

В спектрах с высоким отношением сигнал/шум можно увидеть признаки аккреции в смещенных в красную сторону абсорбционных компонентах фотосферных линий металлов. На рис. 3 приведены 


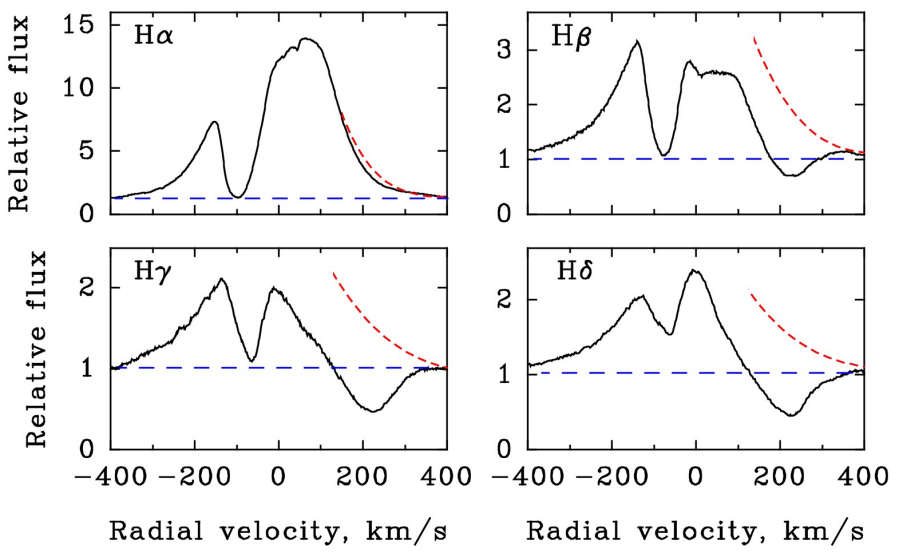

Рис. 2. Бальмеровские линии в спектре S CrA SE. В длинноволновом крыле пунктиром показан участок коротковолнового крыла, симметрично "отраженного" относительно нулевой скорости. Шкала длин волн астроцентрическая
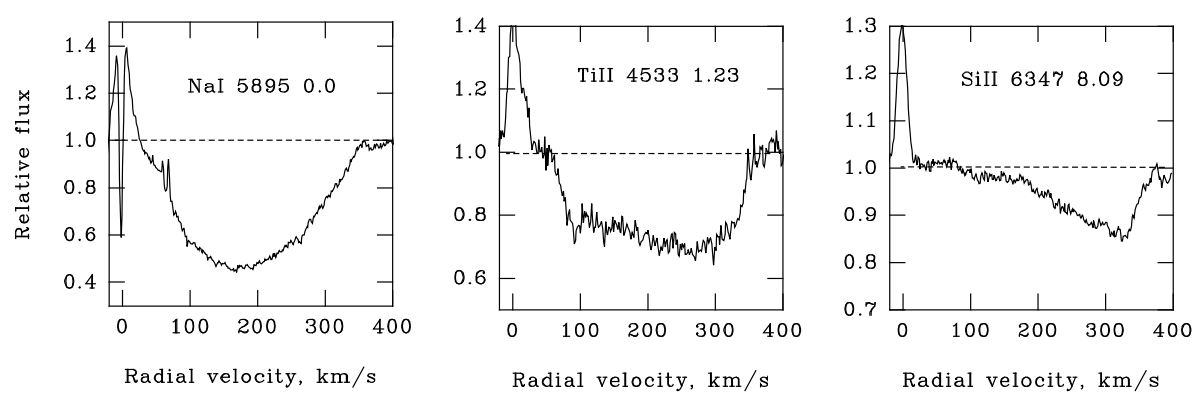

Рис. 3. Профили линий, образующихся в аккреционном потоке S CrA SE

профили линий металлов в спектре S CRA SE. Наибольшую скорость падения показывают линии с высоким потенциалом возбуждения $(8 \mathrm{eV})$, образующиеся у основания аккреционной колонки, вблизи ударной волны, где температура и плотность падающего газа наиболее высоки (Petrov et al., 2014a).

Другим следствием магнитосферной аккреции считается вуалирование фотосферного спектра CTTS. Жесткое излучение ударной волны в основании аккреционного канала ионизует падающий газ, а также прогревает фотосферу звезды до оптической толщи $\tau>1$ в континууме. В результате образуется горячее фотосферное пятно при температуре около 8000 K (Lamzin et al., 1996; Gullbring et al., 2000).

На фоне излучения фотосферы звезды позднего спектрального типа такое пятно дает дополнительный континуум, который уменьшает контраст фотосферного спектра остальной, невозмущенной части фотосферы. В наблюдаемом спектре звезды эквивалентные ширины EW фотосферных линий оказываются уменьшены. Количественным выражением этого эффекта служит так называемый фактор вуалирования (veiling factor) $\mathrm{VF}=\mathrm{EW}(\mathrm{sp}) / \mathrm{EW}(\mathrm{obs})-1$, где $\mathrm{EW}(\mathrm{sp})$ - эквивалентная ширина фотосферной линии, соответствующая спектральному типу невозмущенной фотосферы, а $\mathrm{EW}(\mathrm{obs})$ - наблюдаемая эквивалентная ширина.

Наблюдаемые спектры CTTS показывают факторы вуалирования от нуля до единицы и более. Однако детальное исследование этого эффекта обнаруживает разногласие с простой моделью горячего пятна. Прежде всего, модель предполагает корреляцию вуалирования и блеска звезды. Если фактор вуалирования на длине волны $5500 \AA$ равен единице, то блеск звезды в полосе $\mathrm{V}$ должен быть увеличен на $0.75^{m}$. В 1995-1999 гг. на обсерватории NOT были проведены три серии одновременных спектральных и фотометрических наблюдений RW Aur A (Petrov et al., 2001). Блеск звезды и фактор вуалирования менялись в больших пределах, однако корреляции между ними не было. Возможно, эта корреляция была нивелирована переменной околозвездной экстинкцией. 
Исследование эффекта вуалирования требует высокого спектрального разрешения и высокого отношения сигнал/шум, чтобы можно было измерять эквивалентные ширины самых слабых линий. Такие измерения были проведены в спектрах нескольких CTTS, полученных Дж. Хербигом на телескопе Keck-1 (Rei et al., 2018). На рис. 4 приведены результаты измерения вуалирования большого числа спектральных линий, включая самые слабые с эквивалентной шириной 10 mА. В качестве стандарта соответствующего спектрального типа использовались синтетические спектры, рассчитанные с помощью программного пакета SME (Valenti and Piskunov, 1996). На рис. 4 отношение эквивалентных ширин $\mathrm{EW}(\mathrm{synth}) / \mathrm{EW}(\mathrm{obs})$, равное единице, соответствует VF = 0. Видно, что большинство линий показывают значительный уровень вуалирования, в то время как самые слабые не изменены, то есть горячее фотосферное пятно на поверхности этих звезд очевидно отсутствует.
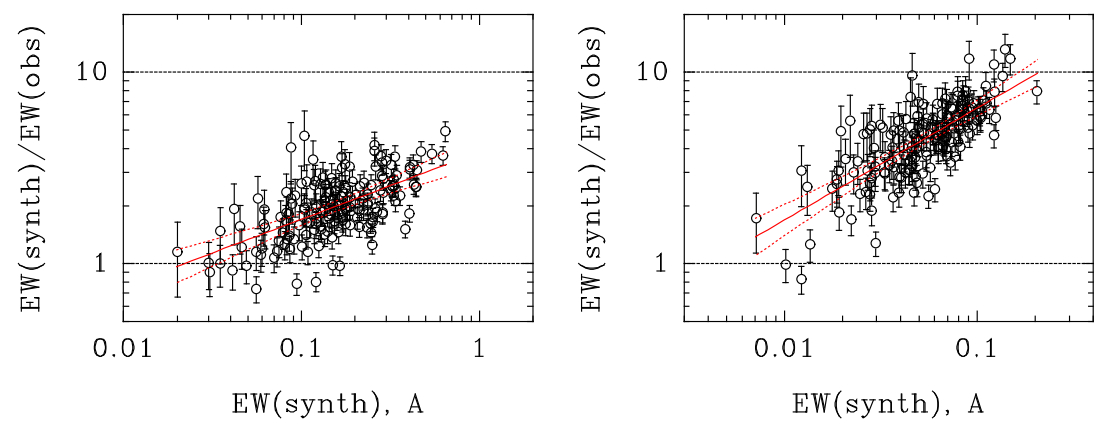

Рис. 4. Отношение эквивалентных ширин фотосферных линий в спектре стандарта и в спектре CTTS: слева LkHa 321 Cyg, справа V1331

В серии спектров DR Tau, полученных на телескопе NOT в 2007 г., были измерены эквивалентные ширины большого числа фотосферных линий разной интенсивности. Так же как и в приведенном выше случае, сильные линии (большей силы осциллятора) оказались более подвержены вуалированию, нежели слабые линии. В течение нескольких ночей наблюдений уровень вуалирования менялся, но указанный эффект сохранялся (Petrov et al., 2011).

В рамках модели магнитосферной аккреции это означает, что аккреция меняет температурный профиль фотосферы: верхние слои нагреваются излучением ударной волны, в то время как нижние остаются неизменными. Возникает "хромосферное пятно". В наблюдаемом спектре звезды фотосферные абсорбции заполняются хромосферной эмиссией, что и вызывает эффект вуалирования. Следует отметить, что подобное объяснение эффекта вуалирования упоминалось еще в самой первой работе Јоу (1945), где описывались спектральные особенности звезд типа Т Тельца.

Область повышенной хромосферной эмиссии, вызванная аккрецией, искажает наблюдаемые профили фотосферных линий. Если "хромосферное пятно" смещено относительно полюса вращения звезды, лучевые скорости, измеренные по фотосферным абсорбциям и по узким эмиссионным линиям, например, Не г $5876 \AA$, меняются в противофазе. Такой эффект наблюдался у нескольких CTTS: DR Tau, DI Cep, RU Lup, RW Aur A, S CrA SE (Petrov et al., 2011).

При усилении потока аккреции прогреваются более глубокие слои фотосферы и образуется "горячее пятно", излучающее в континууме. Процесс нагрева фотосферы CTTS излучением ударной волны и образование эмиссии как в континууме, так и в линиях были рассмотрены в работе Додин, Ламзин (2012).

Хотя горячие пятна, вызванные аккрецией, влияют на видимый блеск звезды, наблюдаемая нерегулярная переменность блеска CTTS часто бывает обусловлена околозвездной экстинкцией. Это хорошо заметно на примере звезды RY Tau, поскольку она видна под большим углом наклона и луч зрения пересекает запыленную атмосферу диска. При изменении блеска звезды в пределах одной звездной величины вуалирование в видимой области спектра не меняется (Babina et al., 2016). Это означает, что причиной переменности блеска является околозвездная экстинкция. Наблюдения указывают на то, что пылевой экран расположен вблизи звезды и представляет собой, по-видимому, запыленный ветер вблизи радиуса сублимации пыли (см. следующий раздел). 


\section{3 Ветер и околозвездная пыль}

Истечение (ветер) происходит за пределами магнитосферы CTTS, где газ течет вдоль открытых силовых линий крупномасштабного магнитного поля. Хотя используется термин "ветер", механизмы истечения CTTS отличны от тех, что ответственны за солнечный ветер или ветер горячих звезд. Несколько типов ветра молодых звезд были предложены и детально рассмотрены в литературе.

Дисковый ветер стартует с протяженной поверхности аккреционного диска и ускоряется магнитной центрифугой: вещество течет вдоль открытых силовых линий магнитного поля диска (Маtt and Pudritz, 2005). X-ветер стартует с границы магнитосферы и диска и ускоряется магнитной центрифугой (Shu et al., 1994). Также на внутренней границе диска может возникать и конический ветер, ускоряемый магнитным давлением азимутальной составляющей магнитного поля на границе магнитосферы (Romanova et al., 2009). Механизм пропеллера возникает в том случае, когда угловая скорость вращения звезды и магнитосферы превышает угловую скорость кеплеровского вращения на внутренней границе диска (Romanova et al., 2018). Это может происходить при ослаблении темпа дисковой аккреции или при усилении магнитного поля звезды. В противном случае, когда внутренний диск вращается быстрее, чем магнитосфера, возникают биполярные магнитосферные выбросъ вещества вследствие раскрытия магнитосферы и перезамыкания силовых линий - магнитосфера постоянно "перестраивается" (Goodson et al., 1997; Zanni and Ferreira, 2013). В полярной области CTTS может также возникать истечение вдоль открытых линий магнитного поля звезды, т. н. полярный ветер (Cranmer, 2008).

Из перечисленных выше механизмов истечения видно, что главной причиной истечения является аккреция - дисковая или магнитосферная. Магнитное поле (диска или звезды) перенаправляет часть аккрецирующего вещества в отток. Таким образом система "звезда + диск" освобождается от избыточного углового момента.

Из наблюдений не всегда можно установить, какой механизм истечения реализуется в каждом конкретном случае. Наблюдатели анализируют профили спектральных линий, уширенных эффектом Доплера, и их переменность во времени. Обсуждение профилей эмиссионных линий в спектрах CTTS в рамках моделей аккреции и ветра можно найти в книге Hartmann (1998). Атлас наблюдаемых профилей $\mathrm{H}_{\alpha}$-эмиссии в спектрах CTTS и НАeBe представлен в статье Reipurt et al. (1996).

Анализируя спектры CTTS, Alencar and Basri (2000) установили, что широкие эмиссионные линии образуются как внутри магнитосферы в потоках падающего газа, так и вне ее, в потоках ветра. Модельные расчеты, выполненные Kwan and Fischer (2011), показали, что широкие эмиссионные линии Н г и Не і в спектрах CTTS образуются в радиальных потоках ветра при электронной плотности $\mathrm{N}_{\mathrm{e}}=10^{9} \mathrm{~cm}^{-1}$ и температуре $\mathrm{T}_{\mathrm{e}} \sim 10^{4} \mathrm{~K}$, а линии Са II - в магнитосферных потоках и на внутренней границе диска при $\mathrm{N}_{\mathrm{e}}=10^{12} \mathrm{~cm}^{-1}$ и $\mathrm{T}_{\mathrm{e}} \leq 7500 \mathrm{~K}$.

Alencar et al. (2005), анализируя спектры RW Aur A, пришли к выводу, что бальмеровские линии образуются в основном в узко коллимированном ветре, стартующем на внутренней границе диска. Более протяженная область дискового ветра хуже объясняет наблюдаемые профили эмиссионных линий.

Модели дискового ветра и магнитосферной аккреции использовались для расчета профилей спектральных линий (см., например, Kurosawa et al., 2011; Dmitriev et al., 2019). Сравнение расчетных профилей с наблюдаемыми позволяет уточнить параметры моделей и оценить темп потери массы. Один из примеров: V1331 Cyg - CTTS, видимая со стороны полюса. Сравнение наблюдаемых профилей бальмеровских линий с рассчитанными в рамках моделей магнитосферы и дискового ветра позволили определить темп потери массы. Показано, что переменность профиля типа Р Cyg на шкале времени в несколько лет вызвана изменениями угла коллимации дискового ветра в пределах нескольких градусов и темпа потери массы в пределах $(6-11) \cdot 10^{-8} \mathrm{M}_{\odot} / \mathrm{yr}$ (Petrov et al., 2014a).

Другой пример: двойная звезда S CrA, состоящая из двух CTTS - S CrA SE и S CrA NW. Mоделирование профилей бальмеровских линий позволило определить, что угол наклона оси вращения каждой звезды составляет 65 градусов (Gahm et al., 2018), в то время как интерферометрические наблюдения в ближнем ИК-диапазоне указывали на малый угол наклона аккреционного диска. Это расхождение вызвано, вероятно, тем, что в наблюдаемом ИК-излучении диска присутствует также тепловое излучение пыли в дисковом ветре (Bans and Koenigl, 2012), что затрудняет определение угла наклона диска.

Интерферометрия CTTS в ближнем ИК-диапазоне показывает, что внутренняя граница пылевого диска (зона сублимации пыли) находится на расстоянии 0.05-0.2 a.e. (Akeson et al., 2005). Это уже 
близко к зоне формирования истечения (магнитосферные выбросы, пропеллерный ветер и др.). Tambovtseva and Grinin (2008) показали, что пыль может существовать в ветре CTTS, и запыленный ветер является причиной околозвездной экстинкции.

Спектральный и фотометрический мониторинг CTTS RY Tau в течение нескольких лет позволил впервые обнаружить, что во время эпизодов интенсивного истечения уменьшалась околозвездная экстинкция, что было интерпретировано как влияние магнитосферных выбросов на запыленный дисковый ветер (Babina et al., 2016). Физический механизм этого явления не вполне ясен. Можно предположить, что горячий газ, выброшенный при раскрытии магнитосферы, "сметает" запыленный дисковый ветер на внутренней границе пылевого диска. В интервалах между такими выбросами дисковый ветер снова поднимет пыль на луч зрения и околозвездная экстинкция восстанавливается. Следует учитывать также, что магнитосферный выброс должен оказывать влияние на магнитное поле диска, что может вносить возмущения в течение ветра на внутренней границе диска. В любом случае найденная зависимость между выбросом газа и изменением околозвездной экстинкции позволяет установить расстояние, на котором находится пылевой экран. Время запаздывания между

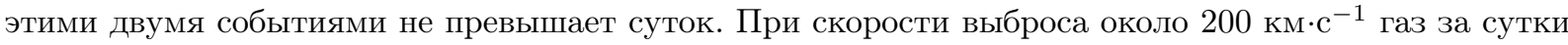
проходит расстояние $\sim 0.1$ а.е., что совпадает с расстоянием сублимации пыли (внутренняя граница пылевого диска). Таким образом, можно предположить, что в случае RY Таu пылевой экран - это горячая пыль в дисковом ветре на расстоянии сублимации, как это схематически показано на рис. 5.

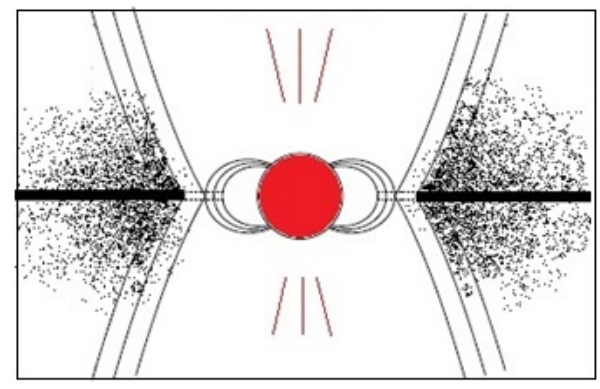

Рис. 5. Запыленный дисковый ветер RY Tau

Вывод о том, что RY Tau видна сквозь запыленную атмосферу внутреннего аккреционного диска, был также получен при анализе интерферометрических наблюдений RY Tau в видимом и ближнем ИK-диапазонах (Davies et al., 2020). Аналогичный результат был получен и для SU Aur: горячая пыль над плоскостью диска является необходимым ингредиентом для объяснения избытка излучения на длине волны $2.2 \mathrm{mkm}$ (Labdon et al., 2019).

Другой пример, показывающий присутствие горячей пыли в дисковом ветре, - двойная система RW Aur. Главный компонент, RW Aur A, - одна из самых ярких и наиболее часто наблюдаемых CTTS. Вторичный, менее массивный компонент, RW Aur B, в среднем на три звездные величины слабее. Компоненты находятся на угловом расстоянии $\approx 1.5$ угловой секунды, что позволяет исследовать их отдельно при хорошем качестве изображения (Antipin et al., 2015). В недавней работе Dodin et al. (2020) было показано, что RW Aur B проявляет характеристики звезды типа UX Ori. Начиная с 2010 г. наблюдались значительные ослабления видимого блеска RW Aur A, достигающие трех звездных величин на шкале времени около года, так что временами главный компонент становился слабее вторичного. Такие глубокие и продолжительные ослабления блеска случались и у других CTTS и интерпретировались как затмения пылевыми облаками. В случае RW Aur A сначала была предложена гипотеза о том, что поглощающая пыль находится на значительном расстоянии от звезды, в приливном газопылевом рукаве, образовавшемся при прохождении вторичного компонента через периастр (Rodriguez et al., 2018). Однако фотометрические наблюдения показали, что при ослаблении потока излучения RW Aur A в видимом диапазоне одновременно возрастал поток на длинах волн 2-5 мкм (Shenavrin et al., 2015). В этом диапазоне излучает пыль вблизи температуры сублимации. Авторы предположили, что ослабления блеска в видимом диапазоне вызваны появлением в дисковом ветре RW Aur А горячей пыли при температуре около 1000 K. Аналогичная интерпретация ранее была предложена Grinin et al. (2009) для объяснения глубокого минимума звезды типа Т Тельца V1184 Таu, где падение блеска в оптическом диапазоне при постоянном или 
слегка увеличившемся излучении в полосе К были вызваны одной причиной: увеличением толщины диска вблизи зоны испарения пыли в результате усиления дискового ветра.

Dodin et al. (2019) зарегистрировали значительное увеличение линейной поляризации RW Aur A во время глубоких минимумов 2010-2011 и 2014-2018 гг. Величина и ориентация вектора поляризации указывали на то, что звезда и внутренние области аккреционного диска были экранированы аксиально-симметричным запыленным дисковым ветром. Спектральные наблюдения (Takami et al., 2016) обнаружили, что во время минимума блеска в спектре RW Aur A почти исчезли все фотосферные линии, за исключением самых сильных. Такое явление было впервые предсказано и смоделировано в работе Гринина, Мицкевича и Тамбовцевой (2006). Во время глубокого минимума блеска звезда закрыта пылью и наблюдается в основном рассеянный свет. При рассеянии излучения звезды движущимися пылинками во внутренних областях околозвездного диска и дискового ветра спектральные линии уширяются вследствие эффекта Доплера. В результате слабые линии становятся размытыми, практически незаметными.

Увеличение на порядок величины содержания железа в короне RW Aur A было зарегистрировано рентгеновской обсерваторией Chandra во время минимума блеска в 2017 г. Было высказано предположение, что разрушение планетозимали или планеты земного типа могло привести к значительному увеличению пыли, которая была аккрецирована на звезду (Günther et al., 2018).

\section{4 Динамика потоков аккреции и ветра}

Профили широких эмиссионных линий в спектре CTTS дают представление о направлении и скорости газовых потоков вокруг звезды. Обзор и классификация профилей эмиссионных линий в спектрах CTTS приведены в статье Reipurt et al. (1996). Анализ профилей эмиссионных линий водорода и металлов в спектрах 30 CTTS выполнен Alencar and Basri (2000). Образование различных типов профиля $\mathrm{H}_{\alpha}$-эмиссии в спектрах CTTS было рассмотрено в рамках моделей магнитосферы и дискового ветра (Kurosawa et al., 2006).

Процессы аккреции и истечения существенно нестационарны. Наблюдения переменности профилей спектральных линий могут дать дополнительную информацию о той среде, где образуются эти линии. Продолжительные серии спектральных наблюдений были выполнены лишь для нескольких CTTS (см. табл. 2)

Таблица 2. Серии спектральных наблюдений CTTS

\begin{tabular}{lcl}
\hline Звезда & Период наблюдений & Ссылка \\
\hline SU Aur & $1986-1990$ & Giampapa et al. (1993) \\
& $1986-1992$ & Johns and Basri (1995) \\
& $1990-1994$ & Petrov et al. (1996) \\
& $2013-2019$ & Petrov et al. (2019) \\
RW Aur A & $1995-1999$ & Petrov et al. (1996) \\
& $1989-1999$ & Alencar et al. (2005) \\
& $2010-2015$ & Takami et al. (2016) \\
DR Tau & $1989-1998$ & Alencar et al. (2001) \\
RY Tau & $2013-2019$ & Babina et al. (2016), Petrov et al. (2019) \\
\hline
\end{tabular}

Звезда SU Aur (G2 III), с массой $2 \mathrm{M}_{\odot}$, занимает промежуточное положение между CTTS и HAeBe. RW Aur A является типичной CTTS и отличается высоким темпом аккреции (White and Ghez, 2001). Обе звезды ориентированы так, что луч зрения пересекает как область ветра, так и область аккреции. При этом, кроме нестационарных процессов аккреции и ветра, можно обнаружить периодические изменения, связанные с осевым вращением звезды или диска. В спектре SU Aur период около трех суток обнаружен в изменениях интенсивности "синего" крыла эмиссии $\mathrm{H}_{\alpha}$, а также в переменности смещенного в красную сторону абсорбционного компонента Н $\beta$. Это было интерпретировано как вращательная модуляция в рамках модели наклоненного магнитного диполя (Giampapa et al., 1993; Johns and Basri, 1995). Аналогичный эффект аксиальной асимметрии аккреции наблюдался и в случае RW Aur: в изменениях лучевой скорости широких эмиссионных линий был обнаружен период 2.77 суток. По-видимому, за время одного оборота звезды с периодом 
5.5 суток на луче зрения проходят два полюса аккреции (Petrov et al., 2001). Период 5.6 суток был обнаружен в изменениях магнитного поля RW Aur A (Dodin et al., 2012).

Продолжительная серия спектральных наблюдений другой CTTS, DR Tau (K7), включает 103 спектра, полученных в 1989-1998 гг. (Alencar et al., 2001). Звезда демонстрирует значительные изменения в профилях эмиссионных линий на разных временных шкалах, при этом не наблюдается какого-либо периода. Вероятно, это связано с ориентацией диска: изображения аккреционных дисков CTTS, получаемые интерферометром ALMA, показывают, что DR Tau видна под малым углом наклона ("pole-on") (Long et al., 2019). Поскольку постоянно виден только один полюс аккреции, вращательная модуляция не проявляется.
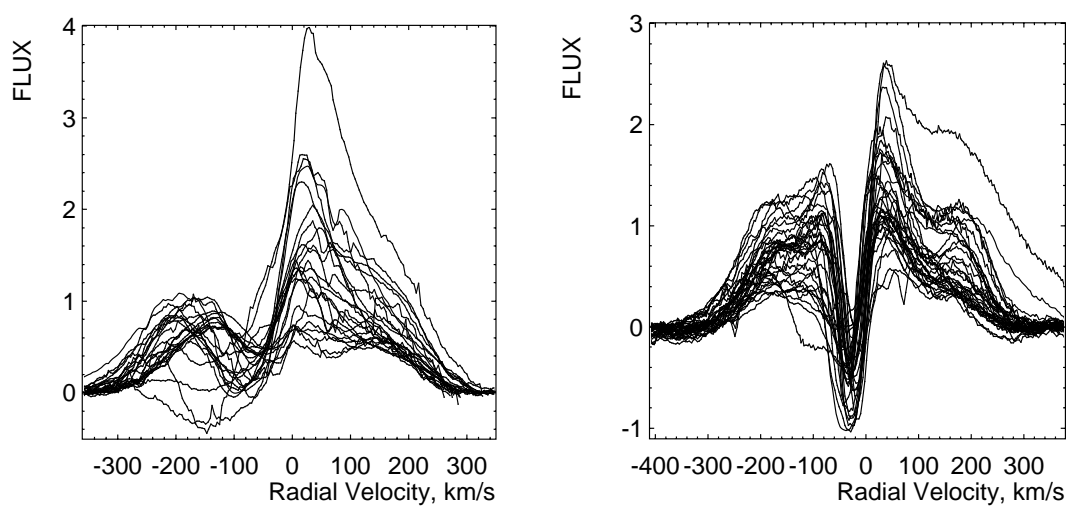

Рис. 6. Диапазон изменений линии $\mathrm{H}_{\alpha}$ в спектре RY Tau (2014-2015 гг.) и в спектре SU Aur (2017-2018 гг.). Поток выражен в единицах $3.67 \cdot 10^{13} \mathrm{erg} \mathrm{cm}^{-2} \mathrm{~s}^{-1} \AA^{-1}$

В КрАО РАН на протяжении нескольких лет проводится спектральный мониторинг двух CTTS RY Tau и SU Aur. Наши спектральные наблюдения сопровождаются одновременной фотометрией в системе BVRI. Это позволяет определить потоки излучения в эмиссионных линиях в абсолютных единицах, что бывает полезным при интерпретации полученных данных. Результаты этого мониторинга за первые пять сезонов (2013-2018) опубликованы в Petrov et al. (2019). В качестве примера на рис. 6 показаны изменения профиля потока в линии $\mathrm{H}_{\alpha}$ в течение одного сезона, когда эти изменения были максимальны. Диапазон изменений линии $\mathrm{H}_{\alpha}$ у обеих звезд заметно меняется от сезона к сезону.
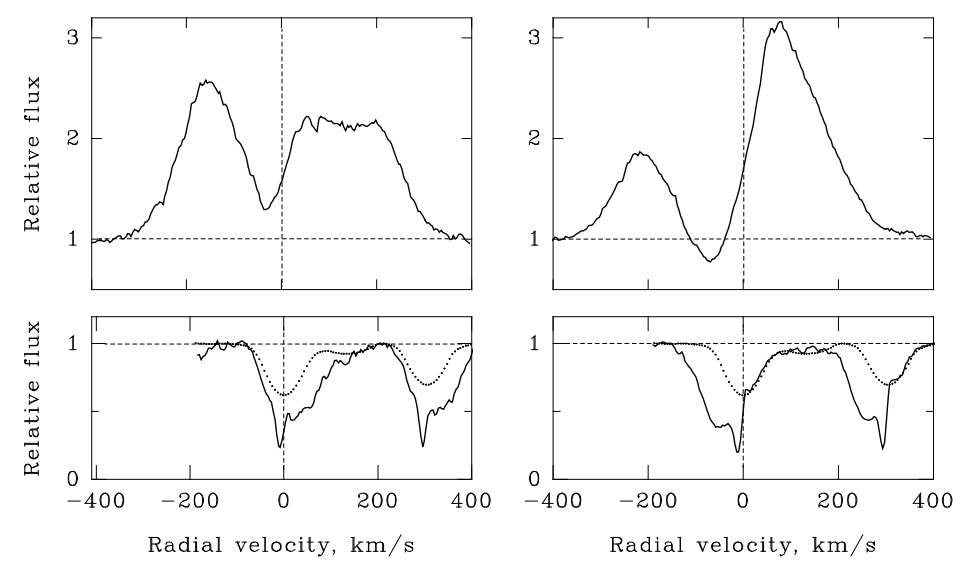

Рис. 7. Профили $\mathrm{H}_{\alpha}$ и D Na I в спектре RY Tau (HJD 2458737 и 2458765). В линиях D Na I пунктиром показан фотосферный профиль звезды G2 III, уширенный вращением звезды, v $\sin i=50$ км/с. Шкала лучевых скоростей - астроцентрическая

B профиле линии поглощения резонансного дублета D Na I в спектре RY Tau хорошо видны переменные абсорбции с красной и с синей стороны от фотосферной линии, возникающие в падающем 
и в истекающем потоках газа (см. рис. 7). Изменения этих абсорбций коррелируют с изменениями в профиле эмиссионной линии $\mathrm{H}_{\alpha}$.

Характерное время изменения абсорбций D NaI, образующихся в падающем и истекающем потоках, составляет около 20 суток. Кроме того, наблюдается изменение средней интенсивности этих абсорбций на протяжении нескольких лет (рис. 8). Возможно, это отражает изменение относительной роли аккреции и истечения.

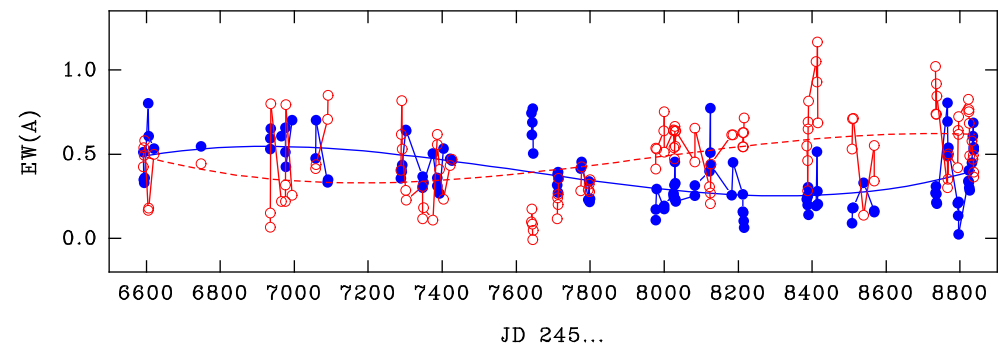

Рис. 8. Долговременные вариации аккреции и ветра RY Tau: изменение эквивалентной ширины абсорбций D Na I, образующихся в потоках аккреции (открытые кружки) и ветра (заполненные кружки) в течение семи лет. Эквивалентная ширина абсорбций измерялась в диапазонах лучевых скоростей $-160 \ldots-30$ км/с (ветер) и $+10 \ldots+150 \mathrm{kм} / \mathrm{c}$ (аккреция). Сплошная и пунктирная кривые - аппроксимации полиномом 3-й степени

Другая звезда, SU Aur, также показывает значительные изменения дисперсии потока излучения в линии $\mathrm{H}_{\alpha}$ от сезона к сезону. В профиле D NaI признаки аккреции почти отсутствуют, в то время как признаки ветра отчетливо выражены в виде глубокой абсорбции на лучевой скорости около $-50 \mathrm{kм} / \mathrm{c}$. Глубина этой абсорбции является индикатором плотности ветра на луче зрения. За время с сентября по ноябрь 2016 г. эта абсорбция исчезла полностью и не появлялась до конца сезона наблюдений (февраль 2017 г.). На рис. 9 показаны несколько совмещенных спектров SU Aur в области D2 Na I. Широкий профиль принадлежит фотосферной абсорбции, уширенной вращением звезды, $\mathrm{v} \sin i=60 \mathrm{kм} / \mathrm{c}$. Более узкая абсорбция переменной интенсивности, образующаяся в ветре на лучевой скорости около $-50 \mathrm{kм} / \mathrm{c}$, отсутствует на спектрах, полученных после 20 ноября 2016 г. В то же время значительно ослабла и аналогичная абсорбция в линии $\mathrm{H}_{\alpha}$. В начале следующего сезона признаки ветра снова были видны.

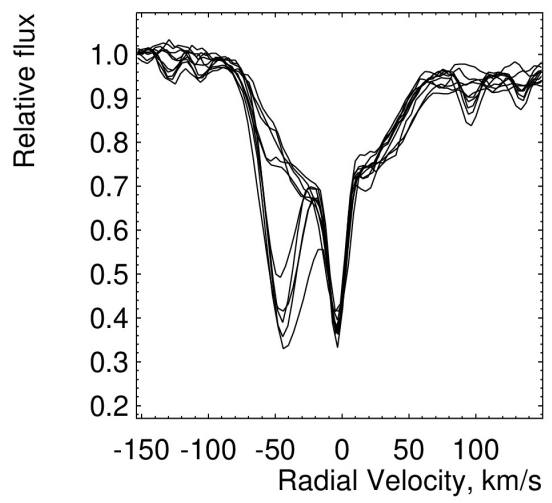

Рис. 9. Изменения профиля линии D2 Na i в спектре SU Aur (сентябрь - ноябрь 2016 г.). Шкала лучевой скорости - астроцентрическая. Абсорбционный пик около нулевой скорости - межзвездное поглощение. Переменная абсорбционная деталь на лучевой скорости около -50 км/с исчезла после 20 ноября 2016 г.

Столь быстрое исчезновение признаков ветра указывает на короткую шкалу расстояний. Повидимому, изменения произошли не в дисковом ветре, стартующем с протяженной поверхности диска. Скорее всего, прекратилось истечение на границе магнитосферы - пропеллерный ветер (см. ниже), что могло быть вызвано как уменьшением темпа аккреции, так и ослаблением магнитного поля звезды. 
Интерпретация наблюдаемых изменений аккреции и ветра CTTS стала возможной после развития методов численного моделирования магнитогидродинамических процессов (см. обзор Romanova and Owocki, 2015). Основные параметры, определяющие режимы аккреции и ветра: масса звезды M, угловая скорость вращения звезды $\Omega$, магнитное поле звезды (магнитный момент диполя $\mu$ ) и темп аккреции массы $\dot{M}$. Отсюда определяются радиус коротации $\mathrm{R}_{\text {со }}{ }^{3}=\mathrm{GM} / \Omega^{2}$ и радиус магнитосферы $\mathrm{R}_{\mathrm{m}}=\mu^{4 / 7} /\left(\dot{\mathrm{M}}^{2} \cdot \mathrm{GM}_{*}\right)^{1 / 7}$. Условие $\mathrm{R}_{\mathrm{co}}=\mathrm{R}_{\mathrm{m}}$ определяет границу между режимом аккреции и режимом пропеллера (Romanova and Owocki, 2015).

Магнитосферная аккреция $\left(\mathrm{R}_{\mathrm{m}}<\mathrm{R}_{\mathrm{co}}\right)$ существенно нестабильна: аккреционный диск "закручивает" магнитосферу, циклически происходит раскрытие и перезамыкание силовых линий, что сопровождается "магнитосферными выбросами" (Goodson et al., 1997; Zanni and Ferreira, 2013). В режиме пропеллера $\left(\mathrm{R}_{\mathrm{m}}>\mathrm{R}_{\mathrm{co}}\right)$ центробежный барьер препятствует аккреции, возникает отток вещества, “пропеллерный ветер", ускоряемый магнитной центрифугой вращающейся магнитосферы. Однако детальное исследование режима пропеллера показало, что эпизодическая аккреция возможна даже в режиме сильного пропеллера (Romanova et al., 2018). На короткие промежутки времени диск может приближаться к звезде, поэтому аккреция становится возможной на границе магнитосферы выше или ниже плоскости диска. Характерное время повторения таких эпизодов аккреции составляет десятки суток. Таким образом, истечение и аккреция в режиме пропеллера также нестабильны.

Звезды RY Tau и SU Aur относятся к быстровращающимся CTTS (v sin $i \approx 50$ и 60 км/с соответственно) и поэтому могут находиться в режиме пропеллера, тем более что RY Tau имеет хорошо видимый джет. В режиме пропеллера относительное количество вещества, выброшенного в ветер, и его скорость увеличиваются с увеличением отношения $\Omega_{*} / \Omega_{K}\left(\mathrm{R}_{\mathrm{m}}\right)$, где $\Omega_{*}$ - угловая скорость вращения звезды и $\Omega_{K}\left(\mathrm{R}_{\mathrm{m}}\right)$ - угловая скорость кеплеровского вращения диска на границе магнитосферы (Romanova et al., 2018). Это означает, что усиление магнитного диполя $\mu$ (увеличение радиуса магнитосферы $\mathrm{R}_{\mathrm{m}}$ ) может быть замечено наблюдателем как увеличение скорости ветра и потока излучения в линии $\mathrm{H}_{\alpha}$.

Звезды RY Tau и SU Aur находятся на радиативном эволюционном треке, то есть имеют радиативное ядро и конвективную оболочку. В случае обнаружения циклических изменений в динамике аккреции и ветра с характерным временем в несколько лет (см. рис. 8) можно предполагать, что причиной таких изменений является магнитная активность молодой звезды. Кроме того, циклические изменения в темпе аккреции вещества на молодую звезду могут быть вызваны также присутствием планеты вблизи магнитосферы (Teyssandier and Lai, 2020), как это наблюдается у CTTS CI Tau (Johns-Krull et al., 2016).

\section{5 Заключение}

Аккреция, ветер и пыль - три фактора, определяющие наблюдаемую активность молодых звезд. Основным источником активности является аккреция. Дисковая аккреция поставляет вещество аккреционного диска на границу магнитосферы звезды. Запыленный дисковый ветер является ответственным за околозвездную экстинкцию и нерегулярную переменность CTTS. Магнитосфера звезды контролирует перенос вещества с внутренней границы аккреционного диска на поверхность звезды, а также вынос части вещества в ветер. Изменения магнитного поля звезды отражаются на динамике аккреции и ветра. Мониторинг аккреции и ветра может выявить скрытые процессы - цикл магнитной активности молодой звезды или орбитальное движение планеты.

Благодарности. Спектральные наблюдения RY Tau и SU Aur на телескопе ЗТШ в 2019-2021 гг., включая обработку и анализ полученных спектров, были поддержаны грантом РНФ 19-72-10063. Автор выражает благодарность С.А. Артеменко, Е.В. Бабиной, К.Н. Гранкину и С.Ю. Горда за многолетнее плодотворное сотрудничество.

\section{Литература}

Амбарцумян В.А., 1949. Астрон. журн. T. 26. С. 3. [Ambartsumyan V.A., 1949. Astron. zhurn., vol. 26, p. 3. (In Russ.)]

Додин А.В., Ламзин С.А., 2012. Письма в Астрон. журн. Т. 38. № 10. С. 1. [Dodin A.V., Lamzin S.A., 2012. Pis'ma v Astron. zhurn., vol. 38, no. 10, p. 1. (In Russ.)] 
Alencar S.H.P. and Basri G., 2000. Astron. J., vol. 119, p. 1881.

Alencar S.H.P., Basri G., Hartmann L., Calvet N., 2005. Astron. Astrophys., vol. 440, p. 595.

Alencar S.H.P., Johns-Krull C.M., Basri G., 2001. Astron. J., vol. 122, p. 3335.

Akeson R.L., Walker C.H., Wood K., et al., 2005. Astrophys. J., vol. 622, p. 440.

Antipin S., Belinski A., Cherepashchuk A., et al., 2015. Inform. Bull. Var. Stars, vol. 6126, p. 1.

Babina E.V., Artemenko S.A., Petrov P.P., 2016. Astron. Lett., vol. 42(3), p. 193.

Bans A. and Koenigl A., 2012. Astrophys. J., vol. 758, p. 100.

Bouvier J., Alencar S.H.P., Harries T.J., et al., 2007. Protostars and Planets V, pp. 479-494.

Camenzind M., 1990. Rev. Modern Astron., vol. 3, p. 234.

Cranmer S.R., 2008. Astrophys. J., vol. 689, no. 1, pp. 316-334.

Davies C.L., Kraus S., Harries T.J., et al. 2020. Astrophys. J., vol. 897, p. 31.

Dodin A., Grankin K., Lamzin S., et al., 2019. Mon. Not. Roy. Astron. Soc., vol. 482, p. 5524.

Dodin A.V., Lamzin S.A., Chuntonov G.A., 2012. Astron. Lett., vol. 38, p. 167.

Dodin A., Lamzin S., Petrov P., et al., 2020. Mon. Not. Roy. Astron. Soc., vol. 497, p. 4322.

Dong R., Najita J.R., Brittain S., 2018. Astrophys. J., vol. 862, p. 103.

Dmitriev D.V., Grinin V.P., Katysheva N.A., 2019. Astron. Lett., vol. 45, no. 6, p. 371.

Folha D.F.M., Emerson J.P., 2001. Astron. Astrophys., vol. 365, p. 90.

Gahm G.F., Petrov P.P., Tambovsteva L.V., et al., 2018. Astron. Astrophys., vol. 614, p. 117.

Ghosh P., Lamb F.K., 1979. Astrophys. J., vol. 234, p. 296.

Giampapa M.S., Basri G.S., Johns C.M., Imhoff C., 1993. Astrophys. J. Suppl. Ser., vol. 89, p. 321.

Goodson A.P., Winglee R.M., Böhm, K.-H., 1997. Astrophys. J., vol. 489, p. 199.

Grinin V.P., Mitskevich A.S., Tambovtseva L.V., 2006. Astron. Lett., vol. 32, p. 110.

Grinin V.P., Arkharov A.A., Barsunova O.Yu., Sergeev S.G., Tambovtseva L.V., 2009. Astron. Lett., vol. 35, p. 114.

Gullbring E., Calvet N., Muzerolle J., Hartmann L., 2000. Astrophys. J., vol. 544, p. 927.

Günther H.M., Birnstiel T., Huenemoerder D.P., et al., 2018. Astron. J., vol. 156, p. 56.

Hartmann L., 1998. Accretion processes in star formation. New York : Cambridge University Press.

Hartmann L., Herczeg G., Calvet N., 2016. Ann. Rev. Astron. Astrophys., vol. 54, p. 135.

Herbig G.H., Bell K.R., 1988. Third Catalog of Emission-Line Stars of the Orion Population. Lick Observatory Bulletin No. 1111, Santa Cruz: Lick Observatory, Ca.

Johns C. and Basri G., 1995. Astron. J., vol. 109, p. 2800.

Johns-Krull C.M., McLane J.N., et al., 2016. Astrophys. J., vol. 26, p. 206.

Joy A.H., 1945. Astrophys. J., vol. 102, p. 168.

Koenigl A., 1991. Astrophys. J., vol. 370, p. L39.

Kurosawa R., Harries T.J., Symington N.H., 2006. Mon. Not. Roy. Astron. Soc., vol. 370, p. 580.

Kurosawa R., Romanova M.M., Harries T.J., 2011 Mon. Not. Roy. Astron. Soc., vol. 416, p. 2623.

Kwan J. and Fischer W., 2011. Mon. Not. Roy. Astron. Soc., vol. 411, p. 2383.

Labdon A., Kraus S., Davies C.L., et al., 2019. Astron. Astrophys., vol. 627, p. 36.

Lamzin S.A., Bisnovatyi-Kogan G.S., Errico L., et al., 1996. Astron. Astrophys., vol. 306, p. 877.

Long F., Herczeg G.J., Harsono D., et al., 2019. Astrophys. J., vol. 882, p. 49L.

Matt S. and Pudritz R.E., 2005. Astrophys. J., vol. 632, p. 135.

Petrov P.P., 2003. Astrophysics, vol. 46, p. 506.

Petrov P.P., Gahm G.F., Gameiro J.F., et al., 2001. Astron. Astrophys., vol. 369, p. 993.

Petrov P.P., Gahm G.F., Herczeg G.J., et al., 2014b. Astron. Astrophys., vol. 568, p. L10.

Petrov P.P., Gahm G.F., Stempels H.C., et al., 2011. Astron. Astrophys., vol. 535, p. 6.

Petrov P.P., Grankin K.N., Gameiro J.F., et al., 2019. Mon. Not. Roy. Astron. Soc., vol. 483, p. 132.

Petrov P.P., Gullbring E., Ilyin I., et al., 1996. Astron. Astrophys., vol. 314, p. 821.

Petrov P.P., Kurosawa R., Romanova M.M., et al., 2014a. Mon. Not. Roy. Astron. Soc., vol. 442, p. 3643.

Pevtsov A.A., Fisher G.H., Acton L.W., et al., 2003. Astrophys. J., vol. 598, p. 1387.

Rei A.C.S., Petrov P.P., Gameiro J.F., 2018. Astron. Astrophys., vol. 610, p. A40.

Reipurt B., Pedrosa A., Lago M.T.V.T., 1996. Astron. Astrophys. Special Suppl. Ser., vol. 120, p. 229.

Rodriguez J.E., Loomis R., Cabrit S.E, et al., 2018. Astrophys. J., vol. 859, p. 150.

Romanova M.M. and Owocki S.P., 2015. Space Sci. Rev., vol. 191, p.339.

Romanova M.M., Blinova A.A., Ustyugova G.V., et al., 2018. New Astronomy, vol. 62, pp. 94-114. 
Romanova M.M., Ustyugova G.V., Koldoba A.V., Lovelace R.V.E., 2009. Mon. Not. Roy. Astron. Soc., vol. 399, p. 1802.

Shenavrin V.I., Petrov P.P., Grankin K.N., 2015. Inform. Bull. Var. Stars, vol. 6143, p. 1.

Shu F., Najita J., Ostriker E., et al., 1994. Astrophys. J., vol. 429, p. 781.

Takami M., Wei Y.-J., Chou M.-Y., et al., 2016. Astrophys. J., vol. 820, p. 139.

Tambovtseva L.V. and Grinin V.P., 2008. Astron. Lett., vol. 34, p. 231.

Teyssandier J. and Lai D., 2020. Mon. Not. Roy. Astron. Soc., vol. 495, p. 3920.

Valenti J.A. and Piskunov N., 1996. Astron. Astrophys. Suppl. Ser., vol. 118, p. 595.

White R.J. and Ghez A.M., 2001. Astrophys. J., vol. 556, p. 265.

Zanni C. and Ferreira J., 2013. Astron. Astrophys., vol. 550, p. A99. 


\title{
Classical T Tauri stars: accretion, wind, dust
}

\section{P.P. Petrov}

Crimean Astrophysical Observatory, Nauchny, 298409

petrov@craocrimea.ru

\begin{abstract}
Classical T Tauri stars (CTTS) are at the early stage of evolution, when the processes of planet formation take place in the surrounding accretion disks. Most of the observed activity in CTTS is due to magnetospheric accretion and wind outflows. Observations of the accreting gas flows and appearance of the line-dependent veiling of the photospheric spectrum in CTTS are considered. Evidence for the dusty wind causing the observed irregular variability of CTTS is presented. Photometric and spectroscopic monitoring of two CTTS, RY Tau and SU Aur, has been carried out at the Crimean Astrophysical Observatory since 2013 aimed at studying the dynamics of accretion and wind flows on time scales from days to years. The observed variations in the dynamics parameters may be caused by changes in the accretion rate and in the global magnetic fields of CTTS.
\end{abstract}

Key words: young stars, RY Tau, SU Aur, RW Aur 